\title{
INGENIERÍA EN COMUNICACIÓN SOCIAL DEL DEPORTE, Apuntes hacia un programa de trabajo
}

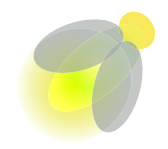

Jesús Galindo Cáceres*

\section{RESUMEN}

Se presenta en un primer momento un apunte de problematización sobre el Deporte considerado como un fenómeno de comunicación social, donde el concepto de comunicación social, desde la perspectiva de una Comunicología sistémica y constructivista, ordena al Deporte como una configuración de sistemas de información y sistemas de comunicación en procesos de difusión-dominación y de interacción-colaboración. Lo cual influye de fondo en la configuración de los procesos sociales generales de convivencia, creación y mantenimiento del mundo social contemporáneo. Jugar fútbol o mirarlo en la televisión, forma y refuerza patrones de competencia o de solidaridad, de individualización o de sentimientos de pertenencia, por ejemplo. En un segundo momento se presenta una síntesis de las ideas expresadas en trabajos anteriores sobre la perspectiva de trabajo de una Ingeniería en Comunicación Social del deporte, desde el contexto de una Ingeniería Social Comunicológica. En un tercer momento se presenta un apunte de programa de trabajo en Ingeniería en Comunicación Social del Deporte, desde el programa tecnológico de la Intervención como estrategia comuniconómica y de construcción socioanalítica.

Palabras Clave: Ingeniería en Comunicación Social, Comunicología, Programa Metodológico, Familias tecnológicas, Intervención, Comunicación Social, Deporte.

\section{SUMARY}

The first stage present a note of questioning on Sport regarded as a phenomenon of social communication, where the concept of social communication, from the perspective of a systemic and constructivist Communicology, directs the sport as a configuration of information systems and systems communication processes of diffusion-domination-collaboration and interaction. This background influences in shaping the general social processes of living, development and maintenance of the contemporary social world. Play football or watch it on television, shape and reinforce patterns of competition and solidarity, identification or feelings of belonging, for example. In a second stage is a synthesis of expressed ideas in previous works about the prospect of working in a Social Communication Engineering of sport, from the context of a social engineering communicological. In a third stage presents a sketch of work program in Communications Engineering of Sport, from the technology program of intervention as a strategy for economic communication and socio-analytical construction.

Keywords: Social Communication Engineering, Communicology, Methodology Program, Families technology, Intervention, Social Communication, Sport.

\footnotetext{
*Mexicano. Doctor en Ciencias Sociales (1985), maestro en Lingüística (1983) y licenciado en comunicación (1978). Autor de 25 libros y más de doscientos ochenta artículos publicados en trece países de América y Europa. Promotor cultural en diversos proyectos desde 1972. Profesor universitario en Argentina, Brasil, Colombia, Perú, España y México desde 1975. Fundador del doctorado en Análisis del Discurso y Semiótica de la Cultura, Escuela Nacional de Antropología e Historia (1996). Fundador del doctorado en Ciencias Sociales, Universidad de Colima (1997). Fundador del doctorado en Comunicación, Universidad

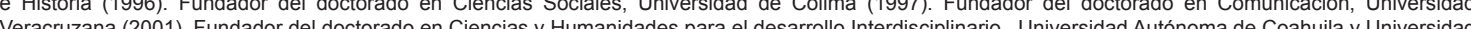
Veracruzana (2001). Fundador del doc). Nacional Autona de Estudios sobre las Culturas Contemporaneas (Programa Culura) desde 1985. Miembro del Sistema Nacional de Investigadores del Consejo Nacional de Ciencia y Tecnología (SNI-CONACYT) desde 1987, SNI III. Promotor del Grupo de Acción en Cultura de Investigación (GACl) desde 1994. Promotor de la Red de estudios en teoría de la comunicación (REDECOM) y del Grupo hacia una Comunicología posible (GUCOM) desde 2003. Miembro de la Red Deporte, Cultura y Sociedad desde 2006. Promotor del Grupo hacia una Ingeniería en Comunicación Social (GINCOM) desde 2009. Miembro del Instituto de Altos Estudios en Deporte, Cultura y Sociedad (INDECUS) desde 2010. arewara@yahoo.com
} 


\section{INTRODUCCIÓN}

El deporte es una parte medular del proceso de civilización, por tanto un proyecto de la modernidad que necesita ajustes y nuevos cauces de promoción en el mundo contemporáneo. El Deporte puede ser considerado como un dispositivo central en los procesos de comunicación de difusión-dominación o de interaccióncolaboración. La práctica deportiva es el primer escenario de la observación científica sobre las situaciones con connotación deportiva. En el otro extremo está la figura del espectáculo. En cualquiera de los puntos de este gradiente de acción percepción se encuentra la tensión termodinámica al centro.

El Deporte excita a la tensión social e individual, de su oportuno manejo operativo depende la configuración de esa tensión y sus consecuencias. Se requieren diagnósticos adecuados científicos de la situación deportiva, y diseños de escenarios deportivos con una nueva imaginación constructiva. Necesitamos entender primero con claridad qué está pasando con el Deporte, y después una decisión sobre lo que queremos hacer con el.

El Deporte es un proyecto social histórico de formación de ciudadanos, es momento de evaluar y rediseñar lo que estamos haciendo con él. En primer lugar se requiere un buen conocimiento tecnológico de toda la metodología a la mano para la exploración y diagnóstico de situaciones deportivas, una buena aproximación científica a la observación-percepción de lo que está sucediendo en la vida social desde una perspectiva comunicológica del Deporte, para construir unos buenos mapas de representaciones sobre lo que sucede, y para obtener buenos diagnósticos de problemas a resolver. $Y$ por otra parte se requiere un buen dominio de la tecnología constructiva de vida social, desde programas metodológicos de Intervención y colaboración de procesos de comunicación social deportiva, para aspirar a diseñar soluciones efectivas de los problemas percibidos en el diagnóstico, dentro contextos bien observados, bien estudiados.

\section{PROBLEMATIZACIÓN DEL DEPORTE COMO FENÓMENO DE COMUNICACIÓN SOCIAL}

Se presenta en este primer apartado un apunte de problematización sobre el deporte considerado como un fenómeno de comunicación social, desde la perspectiva de una Comunicología emergente constructivista, que ordena al deporte como una configuración de sistemas de información y sistemas de comunicación en procesos de difusión-dominación y de interacción-colaboración. Lo cual influye de fondo en la configuración de los procesos sociales generales de convivencia, creación y mantenimiento del mundo social contemporáneo. Por ejemplo, jugar fútbol o mirarlo en la televisión, forma y refuerza patrones de competencia o de solidaridad, de individualización o de sentimientos de pertenencia, de cambio o de reforzamiento de formas de convivencia, entre otras figuras de la vida social. 
El deporte es un fenómeno social que fue adquiriendo importancia y centralidad a lo largo del siglo veinte. Desde la perspectiva de la agenda popular es de los temas más cercanos a la vida cotidiana, sobre todo para los hombres. Es un referente universal, está presente en todas partes del mundo, y el sector más poderoso y consumidor del planeta no podría vivir sin el. Las preguntas sobre lo que significa todo esto están en parte en la mesa de lo interesante y de lo sugerente. Dada su cada vez mayor relevancia económica y política ha ido ascendiendo en los puestos de la agenda general global. En forma paralela a este ascenso la inteligencia y la curiosidad han volteado hacia el poco a poco. Por supuesto primero fueron los negociantes, en cierto sentido los políticos, pero ha sido poca la atención de los intelectuales en términos generales. De alguna forma este texto se ubica en este último sector de la elite mundial, y trata de aportar elementos para ajustar cuentas con el déficit que el mundo académico tiene con tan relevante y aún emergente fenómeno mundial.

Presentar datos que sustenten el argumento de la importancia social, económica y política del deporte es una redundancia, hay ya muchas publicaciones en este sentido. La audiencia del deporte es mayor que la de la audiencia de cualquiera de las grandes religiones. El comité olímpico internacional agrupa más naciones que la ONU, los emblemas corporativos más importantes en muchos países son equipos deportivos o atletas destacados, los deportistas compiten en popularidad con las estrellas del espectáculo y con los líderes políticos y religiosos, el dinero en juego suma cifras astronómicas. $Y$ eso no es todo, al observar e intentar comprender lo que está detrás de todo este inmenso e impresionante mundo deportivo, las hipótesis ponen en juego sentimientos, pasiones, instintos, configuraciones de la vida humana que sólo los paleontólogos, los psicólogos, los antropólogos, habían tenido como foco de su interés. El deporte no aparece en el grupo central de temas universales de la agenda mundial, como el hambre, la educación, el terrorismo, la guerra, el narcotráfico, la contaminación ambiental, la crisis financiera, pero poco a poco se va posicionando como uno de los temas básicos detrás de estos otros. Una carrera por una parte silenciosa, por otra parte casi frívola. ¿Qué es el deporte para el mundo contemporáneo?

Cuando aparece la pregunta por el deporte desde una visión que pretende comprender el asunto más allá de las agendas prácticas de lo inmediato o de la costumbre, todo se complica de inmediato. Un primer recurso es la Historia Social, intentar averiguar desde cuándo nos acompaña, y con ello interrogar a las fuentes sobre cuáles fueron las motivaciones, las razones, las coartadas iniciales de su emergencia, y qué sucedió después, hasta llegar a nuestros días. Este punto es suficiente para agotar la energía de cualquier intención y esfuerzo. Y tiene de momento algunas respuestas, una de ellas la de la Sociología de Norbert Elias, que ubica al deporte como componente central en el proceso de civilización. Para Elias el deporte forma parte del movimiento general en occidente que ensaya la construcción de escenarios y expresiones de la emoción, que en el pasado tuvieron como centro a la guerra y la muerte, en las formas del esfuerzo físico y la psicología de la competencia, pero ahora sin muertes, sin mutilaciones, con ingredientes simbólicos que permiten que las 
formas sociales de la testosterona del mundo pre- moderno se trasformen bajo las nuevas formas del mundo civilizado de la modernidad. Toda una propuesta, toda una visión de comprensión y explicación de la aparición y emergencia del deporte en occidente.

La lectura de Elias no es la única, pero es un buen ejemplo de lo que sucede cuando el deporte es observado más allá de lo evidente. En esta misma línea el proyecto de la Comunicología posible ensaya una visión del deporte desde la perspectiva de la comunicación social. En la propuesta de la Comunicología el deporte es una configuración de sistemas de información y sistemas de comunicación. El fenómeno del deporte construido como asunto de comunicación social permite observar ciertos matices que le dan una profundidad constructiva muy sugerente. Para un primer enfoque desde este punto de vista hace falta un pequeño apunte comunicológico antecedente.

La Comunicología Social posible tiene diversos componentes que pueden aplicarse al fenómeno del deporte. En principio distingue dos dimensiones de composición básica de la vida social desde la relación posible entre sistemas de información en sistemas de comunicación. Un sistema de información en forma elemental es una configuración de instrucciones, de órdenes prescriptitas sobre algo. Así por ejemplo las normas para jugar fútbol son un sistema de información. Pero las normas para jugar fútbol son sólo uno de los sistemas de información que están en juego cuando se juega fútbol, otros sistemas de información también actúan cuando el árbitro pita y el partido inicia. Por ejemplo todo lo relacionado con la mentalidad masculina. Todos estos diversos sistemas de información entran en juego en un sistema de comunicación donde el tipo de relación entre los diversos sistemas de información construye el tipo de sistema de comunicación resultante, uno de los sistemas participantes puede ser el dominante, o pueden llegar a equilibrarse todos los sistemas de información en juego entre sí.

Por ejemplo, el sistema de información de cierto machismo puede ser dominante en cierta región social de hombres que juegan al fútbol. Entonces ellos jugaran guiados sobre todo con un código de macho. Pero si el sistema de información dominante es el de la caballerosidad, entones el juego acontecerá de otra manera. En un tercer tipo de caso, el machismo y la caballerosidad se equilibran para formar un tercer tipo de sistema de comunicación. Este es el tipo de asuntos que la Comunicología observa.

Al mismo tiempo que lo anterior sucede otras configuraciones de información están en juego también. Por ejemplo las personalidades de los jugadores. Cada uno de ellos es un complejo sistema de comunicación donde diversos sistemas de información están conviviendo. Entre los jugadores uno son dominantes sobre otros, el sistema de comunicación del equipo también es una configuración de diversos sistemas de comunicación individuales que en el nivel del equipo pueden ser percibidos con sistemas de información en relación, constituyendo un cierto tipo de sistema de comunicación equipo resultante. Como puede apreciarse la 
figura de sistema de información y sistema de comunicación es conceptualmente relativa a lo que se está observando, y puede configurar diversos sistemas de sistemas en relación.

Las relaciones entre sistemas de información pueden ser de dos tipos básicos, de dominación y de colaboración. En la primera un sistema o grupos de sistemas de información colonizan y dominan a otros, los someten a sus prescripciones, desplazando las de los otros. Por ejemplo en el fútbol, la situación donde la caballerosidad desplace al machismo, o viceversa. En el caso de la colaboración, los sistemas de información no se desplazan o dominan, sino que interactúan como pares y mutuamente se afectan. En el ejemplo presentado sería el caso en que el machismo y la caballerosidad pudieran establecer puntos de negociación, normas de convivencia, de coexistencia, que en lugar de luchar para que sólo uno de los sistemas sobreviva y domine, los dos pudieran en cierto sentido convivir afectándose mutuamente.

Pero está el otro escenario presentado, el de los individuos, además del escenario de los códigos culturales y sociales supra individuales. En este otro escenario los individuos pueden luchar bajo la norma y perspectiva de que uno vence al otro, y que sólo puede haber un vencedor, siempre debe haber un vencido, y que la victoria se justifica con todos los medios y recursos a la mano para lograrla, y es lo único con valor. Pero también pueden colaborar para mutuamente enriquecerse, aportarse elementos que los empoderan en lo individual y en lo grupal, y en donde todos salen beneficiados. Una forma deporte que fomenta sólo la lucha y la dominación es muy distinta a una forma deportiva que fomenta la cooperación y la colaboración.

En el escenario más complejo esta configuración de individuos y de equipos deportivos se relaciona con la otra, la de normas morales y principios constructivos normativos de la acción deportiva y socio-cultural. El resultado es un fenómeno de comunicación que es complejo, y que puede tener diversos matices y configuraciones particulares concretas. Es decir, no todo los juegos de fútbol son iguales, dependen de la construcción de que son objeto por los diversos sistemas de información y comunicación en juego.

Es decir, por una parte es posible la percepción de una configuración diversa de formas de asociación deportiva en un juego como el fútbol. Y por otra parte es posible también observar que en forma tendencial el juego se practica de cierta forma, con ciertos antecedentes y ciertos efectos. El juego refuerza la presencia de ciertos sistemas de información, y lo hace en un acuerdo colectivo en ese sentido. Por ejemplo el machismo. Pero podría ser de otra manera, el mismo juego con ciertos cambios en construcción y configuración de relaciones entre sistemas de información y sistemas de comunicación, podría promover por ejemplo la caballerosidad, la honestidad, el respeto a los otros.

En un principio el fútbol fue sintetizado con esta última pretensión, según la hipótesis de la escuela de Norbert Elias. ¿Qué pasó en el desarrollo de su 
historia? ¿Cómo llegó hasta nuestro ámbito social y cultural latinoamericano y mexicano? ¿Es posible cambiar la historia?

\section{SÍNTESIS DEL CONCEPTO DE INGENIERÍA EN COMUNICACIÓN SOCIAL DEL DEPORTE}

En este segundo apartado se presentará una síntesis de las ideas expresadas en trabajos anteriores sobre la perspectiva de trabajo de una Ingeniería en Comunicación Social del Deporte, en el contexto de una Ingeniería Social Comunicológica. El deporte es una parte medular del proceso de civilización, por tanto un proyecto de la modernidad que necesita ajustes y nuevos cauces de promoción en el mundo contemporáneo. El deporte puede ser considerado como un dispositivo central en los procesos de comunicación social de difusióndominación o de interacción-colaboración. La práctica deportiva en sí misma es el primer escenario de la observación científica sobre las situaciones con connotación deportiva. En el otro extremo está la figura del espectáculo. En cualquiera de los puntos de este gradiente de percepción y acción se encuentra una tensión termodinámica al centro.

El deporte excita a la tensión social colectiva, grupal, e individual. De su oportuno manejo operativo depende la configuración de esa tensión y sus consecuencias. Se requieren diagnósticos adecuados científicos de la situación deportiva, y diseños de escenarios deportivos con una nueva imaginación constructiva. Necesitamos entender primero con claridad qué está pasando con el deporte, y después tomar decisiones adecuadas sobre lo que queremos hacer con el. El deporte es un proyecto social histórico de formación de ciudadanos, es momento de evaluar y rediseñar lo que estamos haciendo con él.

La mirada comunicológica nos permite observar al deporte como un conjunto de prácticas, visiones, juegos de sentido, códigos culturales, objetos, roles, patrones de comportamiento, figuras sentimentales, formas emocionales, expresiones instintivas, mercancías, flujos simbólicos y materiales. El mundo del deporte es un universo. Al sintetizar su complejidad desde la perspectiva de la comunicación, lo que queda es un movimiento que participa de las dos tendencias dinámicas básicas de la acción comunicativa, dominar o colaborar. Este es el primer escenario de la observación comunicológico, a partir de el se configuran otros elementos, se reconstruyen todos los componentes hasta llegar de nuevo a la complejidad original. Ese es el proceso constructivo de un punto de vista, el comunicológico. Pero empecemos desde el principio.

El fenómeno de comunicación social se distingue en un primer momento de observación como parte de alguna de las dos tendencias básicas ya mencionadas. Visto así el deporte aparece como una forma dinámica de los procesos de comunicación para la dominación de un sistema de información sobre otros, o como una forma dinámica de los procesos de comunicación para la colaboración entre diversos sistemas de información. Tomemos de nuevo al fútbol como 
ejemplo. En el campo de juego los jugadores interactúan en una figura de la lucha, la competencia, por el triunfo de un equipo sobre el otro. El observador comunicólogo identifica que los jugadores colaboran hacia dentro del equipo, e intentan dominar al otro equipo. Esto le da al fútbol una configuración compleja, es al mismo tiempo un ejercicio de dominación y un ejercicio de colaboración. Dentro del sentido común contemporáneo de nuestra forma de percibir como aficionados o jugadores del fútbol, afirmaríamos con la Comunicología que un equipo es un sistema de comunicación colaboración, y que un juego oficial es un sistema de comunicación dominación. Esto visto desde la perspectiva normal de un individuo que juega u observa un partido. Pero hay más.

Sobre las figuras anteriores aparecen otras más. El equipo puede estar ordenado como sistema de comunicación dominación. De los once jugadores, la tendencia es a que unos cuantos, a veces uno en especial, dominen a los demás, el resultado es un juego de equipo cargado hacia cierta parte del equipo. Esto se manifiesta en el campo de juego, y en lo que se llama el vestuario, las relaciones personales del grupo de jugadores sin balón de por medio. Por otra parte, para bien del espectáculo, por ejemplo, el que dos equipos colaboren jugando y dejando jugar, beneficia el ir y venir de la bola, y cierto tipo de flujo de acción en el campo de juego. En este segundo escenario básico la visión del juego supone colaboración, no dominación.

El azar tendrá la palabra para definir al ganador en esta competencia abierta de jugar y dejar jugar. La estrategia de juego vigente tiene en el centro otra configuración de comunicación, donde la variante básica es no dejar jugar al otro, o incluso renunciar a jugar abierto para que el otro no juegue con éxito. Como puede apreciarse el sistema de comunicación del equipo y del juego entre dos equipos, es más complejo de lo que parece en un primer lugar. Y si sumamos a otros equipos, ligas, y torneos, la configuración de comunicación se hace más y más compleja, e interesante.

Hasta aquí sólo estamos percibiendo al fútbol desde esta su configuración elemental de equipo y juego. Pero la situación se complica cuando aparecen otros sistemas de información asociados. Por ejemplo, el ethos masculino del macho. El fútbol es un juego que se supone masculino en principio, y promueve formar cierto tipo de hombres, cierto tipo de masculinidad. La masculinidad de la dominación es un tipo muy distinto a la masculinidad de la colaboración. El fútbol es una figura de comunicación que ha reforzado sobre todo la masculinidad de la dominación. La fuerza física, las cualidades físicas del hombre guerrero dominante, son reforzadas por la figura deportiva del fútbol. Es pertinente observar, siguiendo la argumentación y la reconstrucción histórica de Elias, que el fútbol forma aún hoy a guerreros.

La pregunta es qué tipo de guerrero es el que necesita la vida social actual. En la Inglaterra del inicio de la modernidad esta pregunta tuvo una contestación muy clara, el tipo de guerrero necesario era un guerrero que se autocontrolara y permitiera la emergencia de una sociedad más autocontrolada. La alternativa 
ante la que se construye la figura del deporte es la guerra, la violencia, la preponderancia costosísima del más fuerte y agresivo. El deporte nace colaborando con una sociedad emergente, una sociedad donde se pide a los guerreros que sean menos dominantes y más colaboradores, menos agresivos, y más receptivos, menos violentos y más sensibles.

Como puede apreciarse el asunto de fondo con el deporte, con el fútbol en particular, es más complejo que lo que aparece como un simple juego. Por una parte la sociedad requiere cierto tipo de masculinidad, y no la tiene. Por otra parte esa misma sociedad tiene recursos tan valiosos como el deporte para formar esa masculinidad necesaria para una mejor convivencia ciudadana, y no lo aprovecha. Parece ser que tenemos olvidos estructurales. El deporte surge para formar caballeros, ciudadanos, pero la veta de la lucha, de la confrontación, sigue siendo atractiva, y sin la mediación constructiva original, se convierte en una forma más del sólo luchar, del excitarse, del expresar las ganas de matar, de dominar.

El sistema de información general del deporte no ha sido descompuesto hasta niveles por completo retrógradas. Aún en los escenarios más exitosos el deporte es claramente una confrontación alternativa a la lucha hasta morir. El deporte es una lucha que excita en forma similar a la lucha hasta morir, pero sin morir, y sin matar. En todo esto hay configuraciones constructivas muy importantes, poderosas, que pueden seguir la trayectoria actual, comercial y espectacular, hasta dónde esto nos lleve, o pueden ser reordenadas en el programa original de construcción de ciudadanía, de actores modernos. Aquí es en donde puede parece la Ingeniería Social, la Ingeniería en Comunicación Social.

La idea es entonces que lo que hace el deporte lo siga haciendo, pero bajo supervisión de un programa social de construcción de ciudadanía. Como aparece en los párrafos anteriores en buena parte el deporte nace en la modernidad occidental con ese propósito, transformar a los guerreros asesinos, formar ciudadanos. Así que no es algo ajeno o arbitrario el proponerse un programa de ciudadanización a partir del deporte. La Comunicología propone los marcos de observación para entender cómo está siendo construido el deporte en este momento. Y será la Ingeniería en Comunicación Social, la que partiendo de esas observaciones de la Comunicología, proponga rutas de acción para intervenir la práctica deportiva, o la fenomenología deportiva en general, a favor de programas más claramente socio-culturales de evolución ciudadana productiva.

En este programa posible de Ingeniería en Comunicación Social del deporte, los dos escenarios básicos en los cuales se podría aplicar son, por una parte la práctica deportiva en sí misma, afectando al jugador, al deportista, que en su actividad misma dentro de la forma deportiva, se convierte en un ciudadano cada vez más capaz y empático en el proyecto social de formación ciudadana por el deporte. Y por otra parte está el mundo del deporte como espectáculo. Ahí el trabajo es armónico con el primer escenario, pero con otros elementos de intervención. No es lo mismo un deportista que un espectador deportivo, aunque 
están conectados por el mismo dispositivo de la excitación, de la emoción extrema, de la satisfacción por la lucha y la victoria.

La Ingeniería en Comunicación Social del deporte supone un ejercicio de imaginación constructiva superior. Primero se trata de entender lo que pasa hoy con el deporte, conocer a fondo su construcción social y los efectos de esa construcción. Segundo, conocer los elementos constructivos elementales, fundamentales, de la fenomenología del deporte, para entender dónde se encuentran los operadores que construyen lo que tenemos. Tercero, a partir de esos operadores iniciar un proceso de intervención que vaya modificando la fenomenología en la dirección y sentido previstos por el programa de formación de ciudadanos. Y por supuesto en cuarto lugar, el aprendizaje de todo el proceso para ajustar, mejorar, hacer más eficientes y eficaces las operaciones controladas.

El ejercicio de la Ingeniería en Comunicación Social puede ser parte de un programa público, de Estado. Pero también puede ser parte de programas particulares, en escuelas, ligas, asociaciones regionales. Tenemos un espacio de intervención muy grande, el deporte es parte de la vida social contemporánea, una parte sustantiva. Además de hacer crecer todo lo dicho hasta ahora, también hace falta el desarrollo de una propuesta metodológica específica. Esta dimensión del trabajo es el centro operativo de la intervención eficaz de un programa o una acción concertada de Ingeniería en Comunicación Social. De eso trata el siguiente y último apartado de este texto.

\section{HACIA UN PROGRAMA DE INGENIERÍA EN COMUNICACIÓN SOCIAL DEL DEPORTE}

En esta tercera parte se presenta un apunte de programa de trabajo en Ingeniería en Comunicación Social del Deporte, ahora si con mayúsculas, desde el programa metodológico-tecnológico de la Intervención como estrategia comuniconómica y de construcción socioanalítica. En primer lugar se requiere el mejor conocimiento tecnológico posible de toda la metodología disponible para la exploración y el diagnóstico de situaciones deportivas. Con ello se aspira a una buena aproximación científica de la observación-percepción de lo que está sucediendo en la vida social desde una perspectiva comunicológica del Deporte. Y con ello se pretendería construir unos buenos mapas de representaciones sobre lo que sucede, claridad y precisión en el diagnóstico de los problemas por resolver.

Por otra parte se requiere un buen dominio de la tecnología constructiva de la vida social, desde programas metodológicos de Intervención y colaboración en procesos de comunicación social deportiva, para aspirar a diseñar soluciones efectivas a los problemas percibidos en el diagnóstico, siempre dentro de contextos bien observados, bien estudiados.

El punte de arranque para un programa de trabajo en Ingeniería en Comunicación 
Social del Deporte es tener una cierta claridad sobre lo que se va a construir a mediano y largo plazo, desde ciertos parámetros constructivos. Necesitamos algunos conceptos y perspectivas antes de hilvanar lo pertinente. Los primeros conceptos son el de programa de investigación y el de programa metodológico.

Todo se relaciona con el programa de investigación. De ahí parten todos los proyectos particulares, concretos, en el se incuban propuestas e ideas, ahí se acomodan los hallazgos y las preguntas. El programa de investigación tiene marcos macros y micros de desarrollo. En el nivel macro la configuración de preguntas y problemas es general, la totalidad de lo que se quiere conocer o resolver aparece como un gran bloque constituido de pequeños y grandes esfuerzos para desentrañar incógnitas. No es algo que pueda ser construido desde una sola voluntad o un solo lugar, aunque a veces así lo parezca, es más bien algo en lo que muchos concentran su interés y su energía, para entre todos avanzar como un gran conjunto. Los programas de investigación macro son de las dimensiones de los programas generales de una comunidad científica completa, los físicos, los sociólogos, los comunicólogos.

En las proporciones intermedias y micros están los programas de investigación a la escala de regiones de la comunidad científica, de centros de investigación, o incluso equipos o individuos particulares. Aquí lo que es más importante resaltar es el tiempo. El programa de investigación es un objeto del deseo, es algo que se visualiza en el tiempo a largo plazo. Los programas individuales es deseable que se relaciones con los programas de equipos, redes, grupos, y esos programas intermedios a su vez es importante que se relacionen con los grandes programas de la comunidad científica. Todo esto es relativo a lo que los investigadores individuales estén haciendo en particular, y de cómo eso particular se asocia a intereses, preguntas, proyectos, más generales y colectivos. Un mundo de la ciencia ideal frente a un mundo de la ciencia real que trata de alcanzarlo.

El centro de los programas de investigación son las preguntas y los problemas. Las primeras promueven a la ciencia, al conocimiento del mundo y su composición y organización. Los segundos gestionan a la ingeniería, al conocimiento práctico, a la acción reflexiva que resuelve, inventa, crea, lo que necesitamos para mejor vivir y convivir. Es posible entonces hablar de programas de investigación mixtos, y programas de investigación más inclinados a uno y otro énfasis. El sólo responder preguntas o el sólo resolver problemas. La Comunicología supone un énfasis en las preguntas, la Ingeniería en Comunicación Social un énfasis en los problemas.

Si queremos avanzar en este marco, necesitamos herramientas para construir el conocimiento sobre la vida deportiva, y herramientas para construir a la vida deportiva en sí misma. Para ello requerimos de los programas metodológicos. Cadenas de operaciones relativamente estandarizadas que permiten actuar para buscar la respuesta a una pregunta, configurar las preguntas, identificar problemas, y resolverlos. Los programas metodológicos son algo así como una forma de explorar al mundo para conocerlo, o una forma de conocer al mundo 
para intervenirlo. Así que tenemos programas metodológicos para promover a los programas de investigación científica y a los programas de investigación en ingeniería. En nuestro caso programas metodológicos para promover a la Comunicología del Deporte y a la Ingeniería en Comunicación Social del Deporte.

En el caso de la Ingeniería el programa metodológico general supone dos operaciones generales, el diagnóstico de problemas y el diseño de soluciones. Estas dos macro operaciones adquieren configuraciones específicas dependiendo del campo de trabajo de que se trate, la medicina, la mecánica, la electricidad, la construcción de objetos diversos, la vida social, el deporte. En el diagnóstico, la exploración del objeto de estudio es la clave, y por tanto las operaciones de observación, registro y análisis, constituyen el centro del programa metodológico. En el diseño, la intervención en el objeto de trabajo es la clave, y por tanto las operaciones de representación, modelización, simulación y ejecución, constituyen el centro del programa metodológico.

Así que lo requerido para un buen diagnóstico en asuntos del deporte sería un buen punto de observación, o varios, un primer esquema que permita ordenar las observaciones, organizarlas, sintetizarlas. La configuración espacio tiempo es muy importante. El diagnóstico observa algo en un momento presente, pero necesita ubicarlo en una trayectoria constructiva en el tiempo, en un antes y en un después. Eso permite proveer de las claves para el diseño de intervención en ese proceso, donde lo que se quiere es reforzar una tendencia, diluirla, o mantenerla. En el caso del deporte sería observar lo que sucede en las prácticas deportivas o en el fenómeno del espectáculo deportivo, y a partir de ello decidir qué se quiere mantener, qué se quiere diluir, qué se quiere reforzar, e incluso qué es necesario crear que en el diagnóstico no aparece.

El programa posible en Ingeniería en Comunicación Social del Deporte supone entonces un buen programa diagnóstico, donde la Comunicología es el primer referente para observar a las prácticas deportivas y al espectáculo deportivo. Ahí la clave es aquello de observar al deporte como configuración de sistemas de información y comunicación en procesos dinámicos de dominación o de colaboración, dentro de las formas generales comunicológicas de la difusión y la interacción.

Para lograr este conjunto de observaciones necesarias se requiere tiempo y método. Ahí aparecen las familias tecnológicas de observación dentro de los programas metodológicos tradicionales y heterodoxos, que combinan tecnologías distributivas como la encuesta, fenomenológicas como la etnografía, y estructurales como la entrevista, la historia de vida y los grupos de discusión. A todo ello se aplicarán tecnologías analíticas positivistas, como la estadística, y hermenéuticas, como el análisis del discurso. Lo que se busca es conocer el objeto de estudio, construir una buena representación de el, identificando los elementos que están en juego en los problemas propuestos en un inicio, y las configuraciones problemáticas que surgen a partir del programa diagnóstico. 
La pregunta central en el diagnóstico es sobre un problema que se está identificando en su composición y organización. El trabajo sobre el diagnóstico puede ser la respuesta a una demanda, ese es un primer escenario. Puede ser también resultado de la búsqueda intencional de algo que no es del todo evidente. Puede ser la respuesta a un programa general de reingeniería, de desarrollo, de economía de gasto energético. El programa diagnóstico puede responder a diversos escenarios, y procede en todos los casos de forma similar, y con ajustes según las condiciones del inicio del proceso de investigación, los recursos disponibles, el tiempo, y para mucho ingenieros el punto principal, las expectativas de duración de la solución posible.

Después del diagnóstico sigue el diseño de la solución. En ciencias sociales tenemos tecnologías de trabajo que combinan diagnóstico y diseño en el mismo paquete metodológico. Tal es el caso del socioanálisis y de la investigación acción participativa. En un caso se procede por demanda, en el otro a partir de la intención de intervenir a priori por alguna razón una configuración social. Para el caso del deporte y de la perspectiva de la Ingeniería en Comunicación Social, se requiere aplicar con ajustes estas dos propuestas tecnológicas ya probadas y exitosas, al tiempo que se ensayan nuevas propuestas. En el caso de la comunicación como matriz para concebir y desarrollar el ámbito del deporte, la casuística tiene un lugar privilegiado, como en otros campos. En el tema de la comunicación es la Comuniconomía la propuesta más práctica para la construcción de una Ingeniería en Comunicación Social deportiva a partir del análisis de casos concretos.

La Comuniconomía del Deporte supone la configuración de casos típicos de composición y organización tanto del macro tipo de las prácticas deportivas, como del macro tipo del espectáculo deportivo. Se trata de reunir información suficiente y recurrente para tipificar una correlación estándar entre cierto tipo de problemas y cierto tipo de solución. La Comuniconomía simplifica la casuística proponiéndose armar un grupo de casos típicos, con los cuales un ingeniero pueda en poco tiempo diagnosticar y aplicar una solución, reconociendo en el diagnóstico la consistencia de ciertos indicadores con un modelo de problemas típicos construido previamente, y por tanto la correlación probable con una solución ya tipificada a partir de esos indicadores.

La Ingeniería en Comunicación Social del Deporte por tanto trabaja en principio sobre dos grandes escenarios, las prácticas deportivas y el espectáculo deportivo. Se mueve dentro del programa metodológico general de la Ingeniería, el diagnóstico de problemas y el diseño de soluciones. En particular se alimenta de los programas metodológicos estándar de las ciencias sociales para uno y otro asunto. En el caso del diseño de soluciones se mueve en la lógica que en ciencias sociales nos ha mostrado el trabajo del Socioanálisis y la Investigación Acción Participativa. $Y$ busca estandarizar tipos de problemas con tipos de soluciones en una figura denominada Comuniconomía del Deporte. Todo esto enmarcaría en principio el apunte inicial para un programa de trabajo general sobre Ingeniería en Comunicación Social del Deporte. 


\section{BIBLIOGRAFÍA}

ADAMS, Richard N. (1978) La red de la expansión humana, Ediciones de la casa chata, México.

ALCAIDE Hernández, Francisco (2009) Fútbol. Fenómeno de fenómenos, LID Editorial Empresarial, Salamanca.

ANDER-EGG, Ezequiel (1990) Repensando la investigación-acción-participativa, El ateneo, México.

ANDERSON, Benedict (1993) Comunidades imaginadas, Fondo de Cultura Económica, México.

ANDERSON, Tom (1994) El equipo reflexivo, Gedisa, Barcelona.

BAUMAN, Zygmunt (2008) Comunidad, Siglo XXI editores, Madrid.

BERIAIN, Josetxo y Maya Aguiluz (editores) (2007) Las contradicciones culturales de la modernidad, Anthropos, Universidad Autónoma Metropolitana Ixtapalapa, Universidad Nacional Autónoma de México-CEIICH, Universidad Nacional de Colombia, Barcelona.

BODEN, Margaret (1994) La mente creativa, Editorial Gedisa, Barcelona.

BRAUN, Eliezer (2009) Caos, fractales y cosas raras, Fondo de Cultura Económica, México.

BRUNER, Jerome (1988) Realidad mental y mundos posibles, Gedisa, Barcelona.

CÁCERES, María Dolores (2003) Introducción a la comunicación interpersonal, Síntesis, Madrid.

CAFFAREL, Carmen (compiladora) (1996) El concepto de información en las ciencias naturales y sociales, Universidad Complutense, Madrid.

CIMADEVILLA, Gustavo (2004) Dominios. Crítica a la razón intervencionista, la comunicación y el desarrollo sustentable, Prometeo Libros, Buenos Aires.

COULON, Alain (1988) La etnometodología, Cátedra, Barcelona.

DE KERCKHOVE, Derrick (1999) Inteligencias en conexión, Gedisa, Barcelona.

DÉBORD, G. (1995) La sociedad del espectáculo, La Marca, Buenos Aires.

DÍAZ Larrañaga, Nancy (editora) (2006) Temporalidades, Editorial de la Universidad Nacional de La Plata, La Plata. 
ELIAS, Norbert y Eric Dunning (1995) Deporte y ocio en el proceso de la civilización, Fondo de Cultura Económica, México.

ELSTER, Jon R. C. (1990) Tuercas y tornillos, Gedisa, Barcelona.

GALINDO Cáceres, Luis Jesús (1997) Sabor a ti. Metodología cualitativa en investigación social, Universidad Veracruzana, Xalapa.

GALINDO Cáceres, Luis Jesús (2005) Hacia una Comunicología posible, Universidad Autónoma de San Luis Potosí, San Luis Potosí.

GALINDO Cáceres, Jesús (coordinador) (1998) Técnicas de investigación en sociedad, cultura y comunicación, Addison Wesley-Longman, México.

GERGEN, Kenneth J. (1996) Realidades y relaciones, Paidós, Barcelona.

IBÁÑEZ, Jesús (1985) Del algoritmo al sujeto, Siglo XXI, Madrid.

IBÁÑEZ, Jesús (coordinador) (1998) Nuevos Avances en la Investigación social I y II, Proyecto a, Barcelona.

IBÁÑEZ, Tomás (1994) Psicología social construccionista, Universidad de Guadalajara, Guadalajara.

KATZ, Daniel y Robert L. Kahn (1977) Psicología social de las organizaciones, Trillas, México.

MANERO Brito, Roberto (1992) La novela institucional del socioanálisis, COLOFÓN, México.

MARC, Edmond y Dominique Picard (1992) La interacción social, Paidós, Barcelona.

MARTÍN Serrano, Manuel (2007) Teoría de la comunicación. La comunicación, la vida y la sociedad. McGraw Hill, Madrid.

MEAD, George Herbert (1968) Espíritu, persona y sociedad, Paidós, Buenos Aires.

MOLES, Abraham y Elizabeth Rohmer (1983) Teoría estructural de la comunicación y la sociedad, Trillas, México.

MURCIA Florián, Jorge (1997) Investigar para cambiar, Magisterio, Bogota.

PIAGET, Jean (2005) La equilibración de las estructuras cognitivas: problema central del desarrollo, Siglo XXI, México. 
RESÉNDIZ Núñez, Daniel (2008) El rompecabezas de la ingeniería. Por qué y cómo se transforma el mundo. Fondo de Cultura Económica, México.

RHEINGOLD, Howard (2004) Multitudes Inteligentes. La próxima revolución social, Gedisa, Barcelona.

ROGERS, Everett M. y Rekha Agarwala-Rogers (1980) La comunicación en las organizaciones, McGraw-Hill, México.

SCHNEIDER, Eric D. y Dorion Sagan (2008) La Termodinámica de la vida. Física, cosmología, ecología y evolución, Tusquets, Barcelona.

SLUZKI, Carlos (1996) La red social, Gedisa, Barcelona.

TAPSCOTT, Don y Anthony D. Williams (2007) Wikinomics. La nueva economía de las multitudes inteligentes, Paidós, Barcelona.

VARELA, Francisco (1990) Conocer, Gedisa, Barcelona.

VILLASANTE, Tomás R. (2006) Desbordes creativos. Estilos y estrategias para la transformación social, Los libros de la Catarata, Madrid.

VON FOERSTER, Heinz (1998) Sistémica elemental, EAFIT, Medellín.

WATZLAWICK, Paul et al. (1971) Teoría de la comunicación humana, Tiempo contemporáneo, Buenos Aires. 